\title{
Interfaz cerebro-computador multimodal para procesos de neurorrehabilitación de miembros superiores en pacientes con lesiones de médula espinal: una revisión
}

\author{
Carlos Diego Ferrin Bolaños ${ }^{\psi}$, Humberto Loaiza Correa \\ Universidad del Valle. Santiago de Cali, Colombia
}

Recibido 30 de enero de 2018. Aceptado 30 de julio de 2018

\begin{abstract}
Resumen-El número de trabajos relacionados con Interfaces Cerebro-Computador (BCI, Brain-Computer Interface en inglés) directamente aplicados al proceso de rehabilitación de pacientes con lesiones de médula espinal está en aumento debido a la mejora en las técnicas de procesamiento digital de señales y reconocimiento de patrones que permiten relacionar las señales electroencefalográficas con acciones motoras. Los resultados preliminares de las pruebas de las BCI sobre sujetos reales permiten visualizar en un futuro relativamente cercano la inclusión de este tipo de herramientas en los protocolos de rehabilitación. Sin embargo, hay muchas barreras por resolver, principalmente las relacionadas con el aumento del desempeño y la generación de múltiples comandos naturales mediante interfaces cerebro-computador a partir de electroencefalografía superficial (EEG). En este trabajo se hace una revisión de los más importantes trabajos que muestran la evolución, el estado actual y las oportunidades de investigación alrededor de la temática de interfaces cerebro-computador en procesos de neurorrehabilitación de miembros superiores en pacientes con lesiones medulares.
\end{abstract}

Palabras Clave_Lesión Medular, Interfaz Cerebro-Computador, Electroencefalografía, Revisión

\section{Multimodal Brain-Computer Interface for Supporting Upper Limb Neurorehabilitation Processes in Spinal Cord Injury Patients: A review}

\footnotetext{
Abstract-The number of researches related to rehabilitation processes in spinal cord injury patients using Brain-Computer Interfaces is increasing due to the development of improved digital signal processing and pattern recognition techniques that allows decoding motor actions from electroencephalographic signals. Preliminary results on the application of BCI with real

\% Dirección para correspondencia: carlos.ferrin@correounivalle.edu.co

DOI: https://doi.org/10.24050/19099762.n24.2018.1222
} 
experimental subjects allow to envision a rehabilitation scenario using this kind of technology as part of the therapeutic protocols in a near future. Yet, some problems need to be solved: improve target detection performance and the generation of natural commands by non-invasive brain-computer interfaces based on surface electroencephalography are some of them. In this work, we make a review of the most important researches to exhibit the evolution, the current status, and the research opportunities on the use of brain-computer interfaces for upper limb neurorehabilitation in spinal cord injury patients.

Keywords-Spinal Cord Injury, Brain-Computer Interface, Electroencephalography, Review.

\section{InTERface MUlTimOdal CÉREBro-COMPUTAdOR PARA APOIAR OS PROCESSOS DE NEURONA-REABILITAÇÃO DOS MEMBROS SUPERIORES EM PACIENTES COM LESÃO DA MEDULA ESPINHAL: UMA REVISÃO}

Resumo-O número de trabalhos relacionados com Interfaces Cérebro-Computador (BCI, Brain-Computer Interface en inglés) diretamente aplicado no processo de reabilitação de pacientes com lesões de medula espinal está no aumento e a melhoria nas técnicas de processamento digital de sinais e reconhecimento de patrones que permitam relacionar as seqüências de eletroencefalográficas com ações motoras. Os resultados preliminares das provas de BCI sobre sujeitos reais permitem visualizar em um futuro, mais perto da inclusão deste tipo de ferramentas em protocolos de reabilitação. Sin embargo, feno muitas barreras por resolver, principalmente as relacionadas com o aumento do desempenho e a geração de comandos comandos por meio de interfaces cerebro-computador a partir de electroencefalografia superficial (EEG). No presente trabalho, a empresa tem uma revisão dos mais importantes trabalhos e mostra as evoluções, o estado real e as oportunidades de pesquisa em torno da temática de interfaces cerebro-computador em processos de neurorrehabilitação de supostos superiores en pacientes com lesiones medulares.

Palavras chave—Lesão da medula espinhal, Interface Cérebro-Computador, Electroencefalografia, Revisão.

\section{INTRODUCCIÓN}

un cuando Colombia no posee cifras exactas, Ase reporta que el conflicto armado colombiano y los accidentes de tránsito son las principales causas de lesiones medulares [1], SCI (Spinal Cord Injury), siendo a la vez una de las más altas en el mundo. Entre los tipos de lesiones de médula espinal más comunes en Colombia, se tiene las lesiones cervicales y las torácicas, las cuales pueden conducir a las condiciones de cuadriplejía (parálisis parcial o completa de los miembros superiores e inferiores) y paraplejia (parálisis parcial o completa de los miembros inferiores). Ver Fig. 1.

Las lesiones de médula espinal no solo causan parálisis de las extremidades del cuerpo humano sino que limitan otras funciones como el habla, la respiración, excreción, etc. La restauración de las funciones motoras es de suma importancia para las personas afectadas con lesiones de medula espinal.

No existe una cura definitiva para los pacientes con lesión medular $[2,3]$. Los tratamientos actuales solo mejoran los síntomas y las complicaciones de las lesiones medulares completas [2]. Los implantes de células madres [4] y la regeneración de tejidos [5] intentan reparar los daños producidos en la médula espinal; sin embargo, los estudios han demostrado reconectar las médulas, rotas intencionalmente en roedores, pero estos resultados no se pueden comprobar aun en humanos y/o primates no-humanos [6]. Por el contrario, los enfoques neuromoduladores que se dirigen al tejido neural superviviente han permitido a los sujetos con lesiones medulares completas recuperar la funcionalidad después de un proceso de rehabilitación [7]. Esto ha permitido pensar que en el futuro se puedan desarrollar chips BCI (BrainComputer Interface = Interfaz Cerebro-Computador), ver Fig. 2, que decodifiquen las señales cerebrales y permita crear la estimulación justo por debajo de la lesión y de esta forma crear un puente a través de la lesión medular.

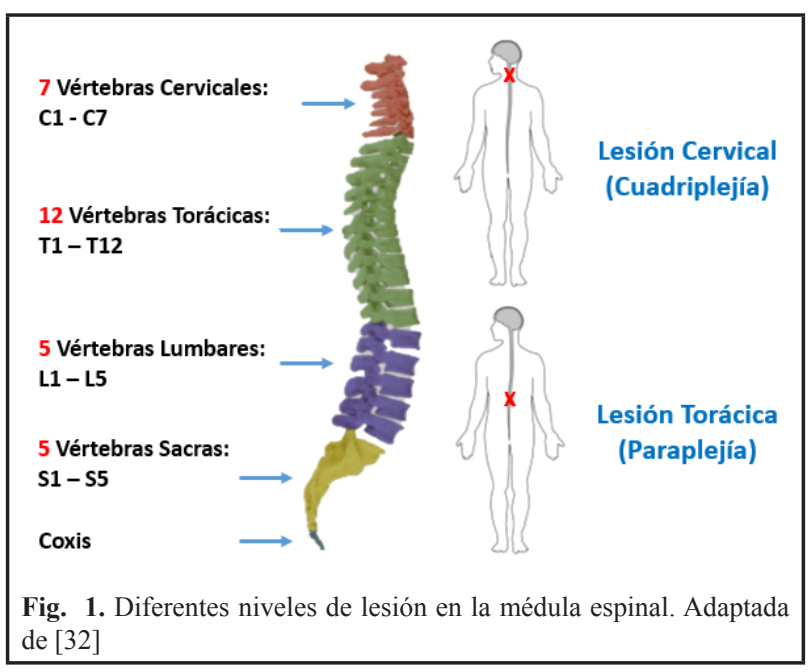




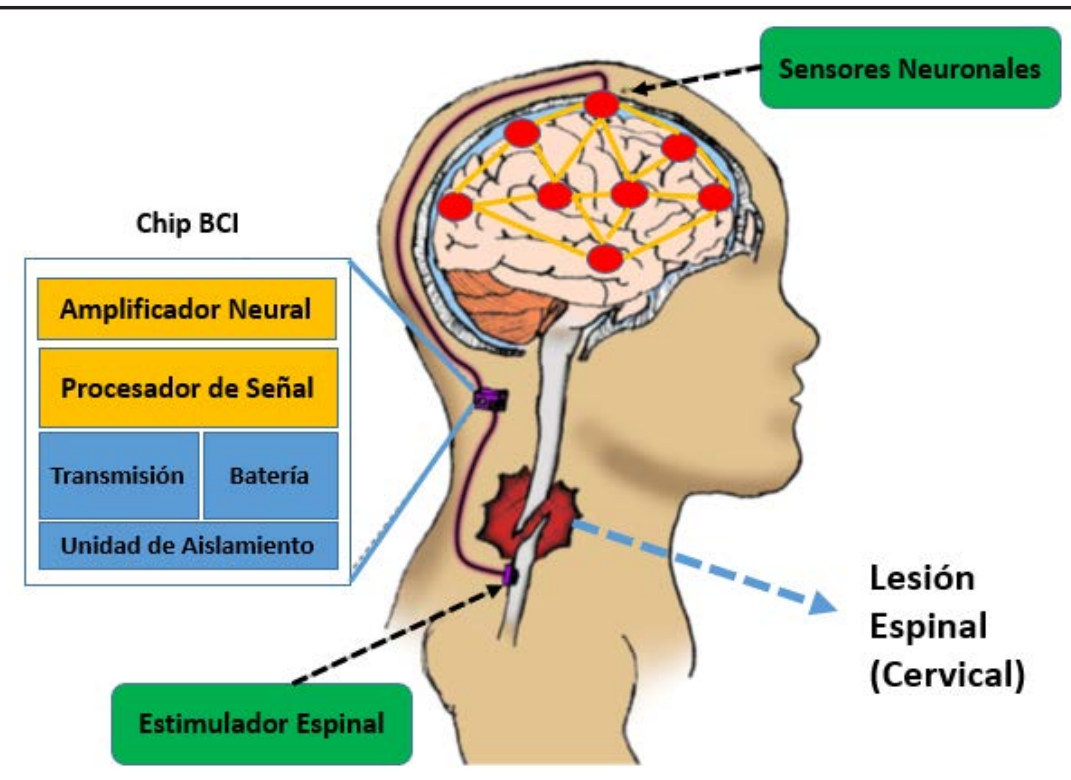

Fig. 2. Chip BCI para neuromodulación. Adaptada de [32]

Una de las grandes inquietudes que surge es si en efecto un proceso de rehabilitación apoyado con $\mathrm{BCI}$ invasiva puede restaurar funciones motoras. $\mathrm{Al}$ respecto, en un caso de estudio realizado en el año 2011 [8] se reporta que un paciente con lesión en C6 logró reducir el tiempo de realización del Test de Minnesota de destreza manual en 1 min y $25 \mathrm{~s}$, demostrándose así que las BCI de imaginación motora pueden ser introducidas en procesos convencionales de rehabilitación a nivel de miembros superiores en pacientes con lesiones medulares. Recientemente, en un reporte científico en la revista Neurosurgery [3] se mostró el efecto de las BCI en procesos de rehabilitación física después de 12 meses de continuo trabajo con ocho pacientes con paraplejía completa, de los cuales 4 pacientes fueron posteriormente diagnosticados con paraplejía incompleta. El grupo de científicos detrás de este proyecto no solo se limitó a mostrar los resultados de tan importante investigación, sino también a plantear los posibles mecanismos que dieron lugar a la rehabilitación debido al uso de la BCI.

En lo siguiente, se expone la evolución, y el estado actual de los procesos de rehabilitación física de miembros superiores en pacientes con lesión medular; igualmente, se describen las interfaces BCI multimodales utilizadas en los actuales procesos de neurorrehabilitación, así como las oportunidades de investigación en este campo.

\section{BCI Multimodales para Pacientes con SCI}

La lesión medular puede definirse como todo proceso patológico (conmoción, contusión, laceración, compresión o sección), de cualquier etiología (traumática y no trau- mática), que afecta la médula espinal, y puede originar alteraciones de la función neurológica por debajo de la lesión: motoras, sensitivas y autonómicas [9]. La complejidad del déficit neurológico, y por tanto del cuadro clínico resultante, depende del nivel y la completitud de la lesión, la extensión transversal o longitudinal del tejido lesionado, y la afectación de sustancia blanca o gris.

Según [1], la SCI tiene grandes consecuencias psicosociales para la persona y su familia, siendo así la principal causante de procesos de discapacidad. La etiología de las lesiones medulares es muy variada e incluye causas de origen congénito, traumático, infeccioso, tumoral o secundario a enfermedades sistémicas. La primera causa de SCI es el trauma, este se encuentra asociado a accidentes de tránsito, heridas por arma de fuego, heridas por arma blanca, caídas de altura, inmersiones en aguas poco profundas, accidentes deportivos y accidentes laborales, entre otras. En segunda instancia se encuentran las lesiones no traumáticas asociadas a factores congénitos, secundarios a diferentes patologías o por intervenciones médicas o quirúrgicas. Este tipo de lesiones ocurren con mayor frecuencia en población joven con una distribución por sexo (hombre:mujer) de 4:1.

La incidencia de las lesiones medulares es variable entre países y entre regiones. En una revisión sistemática de publicaciones científicas entre 1977 y 2006 sobre incidencia, prevalencia y epidemiología de lesión medular a nivel mundial se concluye que la incidencia global oscila entre 10,4 y 83 por millón de habitantes por año, y la prevalencia entre 223 a 755 por millón de habitantes. Se estima que en países desarrollados la incidencia de la lesión medular varía en un rango entre 9 y 53 por millón de habitantes [1]. 
Cada año suceden 12.000 nuevos casos, por ejemplo, en Norteamérica, estimaciones actuales sugieren que la incidencia anual de lesiones medulares en los Estados Unidos es alrededor de 40 casos por millón. En Colombia no se conocen estudios que reporten los datos epidemiológicos de lesión medular. El Instituto de Medicina Legal y Ciencias Forenses estableció que en el año 2005 los accidentes de tránsito habían sido responsables de cerca de 1.036 traumas en las regiones del cuello y del área pélvica, representando el $2,99 \%$ de las personas que presentaron traumatismos en accidentes de tránsito en el país. A su vez, en se indica que la lesión medular es una condición que se presenta en 1 de 40 pacientes colombianos que ingresan a un hospital general consultando por trauma. En un estudio realizado en Bogotá, se encontró que la edad de las personas con lesión medular es en promedio de 35,8 años y en una relación de 4,1:1 (hombre:mujer). La lesión medular se ha reportado como uno de los motivos de consulta más frecuentes en los centros de atención de salud de todos los niveles de complejidad como consecuencia del estado de violencia que vive el país.

Según [1], existen por lo menos cinco tipos de cambios que las personas con lesión medular experimentan con el tiempo: 1) Los efectos de vivir con la lesión por muchos años, tales como deterioro del hombro, infecciones urinarias crónicas o problemas posturales; 2) complicaciones secundarias a la lesión original, tales como siringomielia postraumática; 3 ) procesos patológicos no relacionados con la lesión medular, como enfermedad cardíaca u otras enfermedades crónicas; 4) cambios degenerativos asociados con el envejecimientos, tales como articulares, sensoriales y problemas de tejido conectivo; 5) factores medioambientales, como los sociales, comunitarios y aspectos culturales que pueden complicar la experiencia de envejecer con lesión de la médula espinal.

\section{A. Rehabilitación física de Pacientes con SCI}

La escala más utilizada a nivel internacional para evaluar la extensión o completitud de la lesión medular, es la AIS (ASIA Impairment Scale) [10], que sigue los estándares para clasificación neurológica de la Asociación Americana de Lesiones Medulares ASIA (American Spinal Injury Association). Esta escala clasifica la lesión medular de acuerdo con cinco grados determinados por la ausencia o preservación de la función motora y sensitiva, así: ver Tabla 1.

La mayoría de las lesiones reportadas en las distintas investigaciones a nivel mundial se ubican en los niveles cervical, entre el 34 y 53\% de los casos, y torácico, entre el 43 y $48 \%$, en tanto las lesiones lumbosacras van del 10 a $14 \%$ [1]. La rehabilitación del paciente es un proceso integral: físico (motor-sensitivo), social, psicológico, etc.; en particular la rehabilitación de las capacidades motorassensitivas repercute considerablemente en los aspectos psicológicos y sociales de los pacientes y su familia. En los pacientes con lesiones medulares cervicales que presentan limitada movilidad y maniobrabilidad a nivel de miembros superiores se prioriza la recuperación motriz de estos miembros [11-14]. Esto se debe a que la habilidad de manipular objetos con la mano permite realizar actividades del diario vivir como llevar a la boca alimentos y bebidas esenciales para la supervivencia de cualquier humano.

Dado lo anterior, la atención inicial, el tratamiento, la rehabilitación y la prevención de lesiones en la extremidad superior de las personas con lesiones medulares son de gran importancia para maximizar y mantener la independencia en el paciente. El manejo de la extremidad superior tiende a ser ecléctico e incluye intervenciones de rehabilitación tradicional de entrenamiento dirigido a tareas en las que los pacientes realizan muchas repeticiones de movimientos relevantes para las actividades de la vida diaria, uso de órtesis (félulas y dispositivos de asistencia de miembros superiores $[14,15]$ y la opción de cirugía de extremidad superior, [16]. Según [17], el manejo y la atención de la extremidad superior se pueden dividir en tres fases: la fase aguda, subaguda y reconstructiva. En [18] se propuso una jerarquía similar de restauración funcional de extremidades superiores para individuos con tetraplejía. Esto incluye la provisión de métodos de tratamiento conservadores seguidos de restauración quirúrgica utilizando funciones motoras residuales y la mejora o aumento de las funciones voluntarias con estimulación eléctrica funcional (FES) para la función máxima de la extremidad superior. Los objetivos de las primeras dos fases de la rehabilitación son prevenir complicaciones, lograr un funcionamiento óptimo dentro de los límites del déficit neurológico y crear condiciones óptimas para la fase reconstructiva [19]. En la última fase, varias opciones quirúrgicas y FES están disponibles para mejorar el posicionamiento y la estabilización del brazo, así como la función de agarre fino y palmar [20]. Las nuevas guías de práctica clínica [21] enfatizan la prevención de las lesiones de las extremidades superiores entre individuos con tetraplejia para mantener la independencia.

Aunque no existe un consenso general con respecto al tratamiento de la extremidad superior tetrapléjica, diferentes investigadores, clínicos, y en general consorcios especialistas en el campo de la lesiones medulares [21-23] proporcionan excelentes discusiones y recomendaciones. A pesar de estos, existe acuerdo en que la restauración de la función de la mano es un objetivo importante en la rehabilitación. También vale la pena señalar que existen muy pocas pruebas de extremidades superiores que evalúen con precisión la función motora en esta población; más aún, de 
acuerdo los resultados reportados en [24-26] se encuentra que todavía hay una gran inconsistencia en la evaluación y documentación de la extremidad superior tetrapléjica entre los terapeutas a nivel mundial.

Tabla 1. Escala de Clasificación de la Lesión Medular [10]

\begin{tabular}{cc}
\hline Escala & Descripción \\
\hline Lesión completa A & $\begin{array}{c}\text { Ausencia de función motora y sensitiva } \\
\text { que se extiende hasta los segmentos sacros } \\
\text { S4-S5. }\end{array}$ \\
Lesión completa B & $\begin{array}{c}\text { Preservación de la función sensitiva por } \\
\text { debajo del nivel neurológico de la lesión, } \\
\text { que se extiende hasta los segmentos sacros } \\
\text { S4-S5 y con ausencia de función motora. } \\
\text { Preservación de la función motora por } \\
\text { debajo del nivel neurológico, y más de la } \\
\text { mitad de los músculos llave por debajo } \\
\text { del nivel neurológico tienen un balance } \\
\text { muscular menor de 3. }\end{array}$ \\
& $\begin{array}{c}\text { Preservación de la función motora por } \\
\text { debajo del nivel neurológico, y más de la } \\
\text { mitad de los músculos llave por debajo } \\
\text { del nivel neurológico tienen un balance } \\
\text { Lesión incompleta D }\end{array}$ \\
& Las funciones sensitiva y motora son \\
& normales.
\end{tabular}

El foco principal en la rehabilitación de la persona con lesión medular es la compensación de la pérdida funcional y el uso de aquellas partes del sistema sensomotor [9] que todavía están intactas [24]. Los hallazgos de la investigación sobre neuroplasticidad [27] y recuperación neurológica de la médula espinal también incluyen prácticas de rehabilitación que se centran en estrategias para restaurar la función perdida después de lesión medular, ya que se observa una recuperación significativa de la función después de lesión medular incompleta e incluso completa [28-30]. Existe suficiente evidencia que demuestra y destaca la importancia de comprender las estrategias de control motor que el sistema nervioso central usa para controlar los movimientos de las manos en personas comunes. Esta información puede ser útil para guiar el proceso de rehabilitación después de la lesión medular cervical y garantizar que los ejercicios realizados para la mano y la extremidad superior sean efectivos para restablecer la capacidad funcional [31]. En este sentido la literatura muestra que la rehabilitación de miembros superiores mediante dispositivos de asistencia tecnológica comandadas mediante bioseñales remanentes en los pacientes tales como las EEG, EOG y EMG brindan una gran oportunidad para los terapeutas y pacientes, y constituyen un campo activo de investigación en la actualidad [32, 3, 15, 33-39].

\section{B. BCI multimodales: un nuevo paradigma}

Dado el gran impacto en autonomía que puede proveer las interfaces cerebro-computador [40] en los procesos de rehabilitación [41], la investigación y desarrollo en los últimos años alrededor de este tema ha crecido en forma vertiginosa $[34,40]$.

Una BCI requiere de la adquisición de señales provenientes del cerebro de la persona. Existen en general dos formas de capturar señales cerebrales: los métodos basados en señales metabólicas y los basados en señales eléctricas y magnéticas. Algunas de estas señales cerebrales se encuentran en formato de imágenes volumétricas escalares y otras como series de tiempo en forma de señales multicanales, ver Fig. 3. En los primeros se encuentra la resonancia magnética funcional (fMRI) [42], la tomografía por emisión positrónica [43] y la espectroscopía de infrarrojo cercano funcional (fNIR) [44]. Y en los segundos se encuentra la magnetoencefalografía, la electroencefalografía y la electrocorticografía $[9,45]$.

Estas señales cerebrales son posteriormente procesadas para generar comandos que permiten el control del entorno, la comunicación, el desplazamiento (locomoción), y el comando de dispositivos de asistentica de miembros superiores [15]. Ver Fig. 4. La realimentación es clave en el proceso ya que permite al paciente controlar la BCI hasta que se produzca el efecto deseado.

Muchas de las señales de la Fig. 3 resultan invasivas, poco flexibles y de difícil consecución para desarrollar interfaces cerebro-computador amigables, de bajo costo y portables. Por esta razón se prefieren las BCI basadas en señales superficiales EEG debido a su fácil consecución y no invasividad (Vidaurre, Klauer, Schauer, RamosMurguialday, \& Müller, 2016; Yong \& Menon, 2015). Dentro de los patrones EEG más utilizados para procesos de rehabilitación de miembro superior se encuentran los potenciales corticales relacionados con el movimiento, MRCP (Motor Related Cortical Potential, [48]), dado que permiten la predicción de intención de movimiento [15, 48-50]. El MRCP es un cambio negativo de baja frecuencia en el registro de la señal EEG que tiene lugar aproximadamente 2 segundos antes de la producción del movimiento voluntario. Este a su vez replica los procesos corticales empleados en la planificación y preparación del movimiento.

Existen dos tipos de MRCP: la variación negativa contingente (CNV) [51], y el potencial de preparación (BP, Bereitschaftspotential) [52]. EL CNV se produce junto con la planificación y ejecución del movimiento frente a un estímulo (cue-based), mientras que el potencial de preparación, $\mathrm{BP}$, se genera en respuesta a los movimiento voluntarios, esto es, que no requieren ningún estímulo externo (self-paced). El MRCP se presente tanto en la ejecución de movimientos reales como imaginarios [53]. 

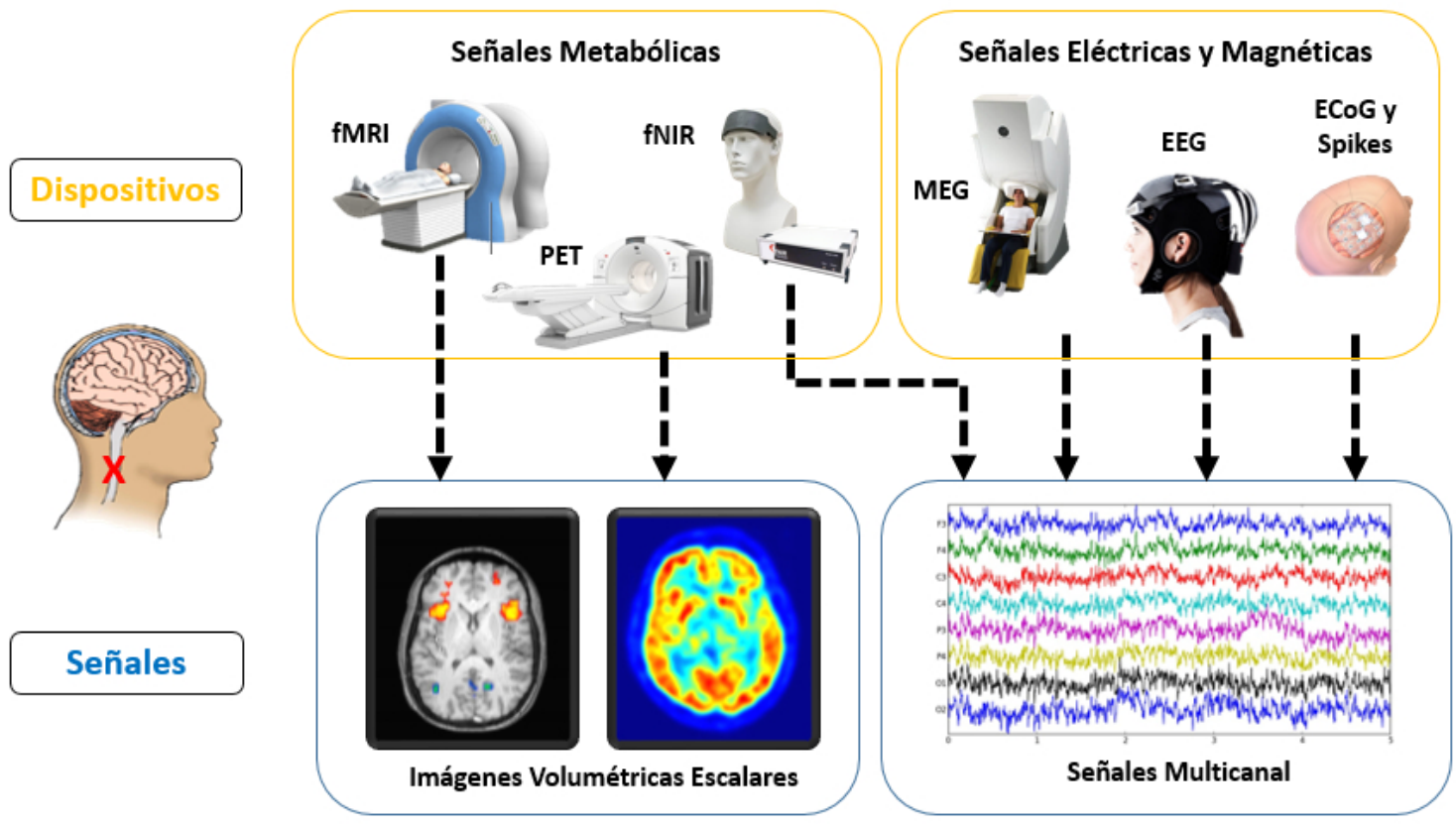

Fig. 3. Técnicas de captura de señales cerebrales: Imágenes Volumétricas y Señales Multicanales

Por lo anteriormente planteado, el MRCP es un patrón muy importante para la identificación auténtica de la intención del movimiento humano, la cual, acompañada del conocimiento de la extremidad involucrada en la ejecución motora y su dirección de movimiento, tiene una implicación potencial en la generación de comandos naturales en dispositivos de asistencia tecnológica de miembros superiores. Esta información puede ser útil en el desarrollo de una herramienta de rehabilitación guiada por el paciente y basada en interfaces cerebro-computadora (BCI). Tal paradigma con un tiempo de respuesta más corto puede resultar más natural para los pacientes con lesiones medulares y también puede inducir plasticidad en el cerebro. En [48] se puede ver una revisión amplia de diferentes trabajos relacionados con BCI basadas en MRCP. Esta revisión permitió evidenciar que a pesar del gran avance que han experimentado las BCI en los últimos años [40], dos retos fundamentales todavía persisten:

1. Mejora del desempeño de detección de patrones cerebrales: a la fecha, son considerables los aportes realizados por diferentes grupos científicos en el mundo para mejorar el desempeño de detección el cual es comúnmente medido en términos del porcentaje de clasificación. Un importante número de BCI con altas tasas de clasificación han sido reportados en la literatura. Por ejemplo en [54] se propuso un método de realimentación basada en los cambios de expresión facial y así mejorar la tasa de clasificación BCI basada en potenciales relacionados a eventos. A su vez, se ha mostrado que diferentes estímulos familiares aumentan los componentes N200 y N400 y así mejoran la tasa de clasificación. Varias técnicas modernas de procesado de señales han sido recientemente desarrollados para $\mathrm{BCI}$, tales como el factorización tensorial, filtros óptimos espaciales, y geometría riemanniana $[55,56]$. Sin embargo, con algunas excepciones, la mayoría de BCI han sido desarrollados para investigación y en su mayoría han sido probado con sujetos sanos. Es muy probable que estos sistemas no se adecuen a personas lesiones de medula espinal ya que hay diferencias significativas en los patrones cerebrales; además, el analfabetismo BCI está presente en un $13 \%$ de las personas con y sin patologías.

2. Generación de comandos naturales: las BCI basadas en estímulos presentan una gran ventaja respecto a las BCI que no utilizan estímulos ya que en las primeras se controla de forma externa cuándo se desea que la BCI opere. Sin embargo, este tipo de comando no es natural ya que el estímulo debe ser controlado por un tercero; por ejemplo, el especialista en un contexto de rehabilitación o la exposición continua al estímulo. Implementar una BCI multimodal que pueda decodificar la intención de movimiento antes que este se produzca es clave para brindar esa sensación de comando natural del dispositivo de asistencia del miembro superior. Muchos trabajos han sido publicados en la literatura científica alrededor de esta problemática [35, 57-64], sin embargo este tipo de $\mathrm{BCI}$ actualmente requieren de largos periodos 
de entrenamiento para lograr un nivel aceptable de naturalidad al comandar el dispositivo de asistencia del miembro superior, esto hace que en la mayoría de los casos pierdan el interés en el uso de este tipo de interfaces, este fenómeno inclusive ocurre en sujetos sanos.

En [65] se plantea la necesidad de crear BCI Multimodales para poder superar estas dos grandes limitaciones de las BCI. Una BCI Multimodal es una BCI más otro sistema interface hombre máquina. Este último puede ser otra BCI basada en otro tipo de patrón cerebral u otro tipo de estímulo. Inclusive puede ser un sistema basado en otro tipo de señal diferente al EEG, por ejemplo, EMG, ECG, EOG, etc. [66-68]. O cualquier interface que permita compartir el control del sistema. La Fig. 5 muestra cuatro clases de BCI Multimodales identificadas en la revisión sistemática realizada por [65].

Los trabajos reportados en $[11,12,35]$ muestran que esta línea de investigación está en un gran crecimiento. Por ejemplo, en la Fig. 6 puede observar un sistema BCI multimodal que utiliza señales EEG y EOG para el control de dispositivos de asistencia de miembros superiores. De igual forma en la Fig. 7 se observa las etapas de una BCI multimodal basada en EEG y EMG, estas últimas son adquiridas en los músculos traseros del cuello ya que algunos personas con SCI pueden generar pequeñas señales que procesadas adecuadamente pueden generar mejores tasas de clasificación para comando de dispositivos de asistencia de miembros superiores o aumentar el número comandos generados naturalmente.

\section{Panorama internacional: 2007 - 2017}

En la Fig. 8 se muestra el número de publicaciones obtenido durante todo el año de 2017. En este tiempo se realizaron búsquedas en bases de datos y diferentes motores de búsqueda bibliográfica tales como EBSCO, Google (Scholar y Patents), Web of Science, Elsevier, IEEE Xplore, Springer, Science Open, etc. Se encontró que estas publicaciones pueden ser organizadas en 4 categorías, a saber, Artículos/Libros, Datasets, Proyecto Colaborativos y Patentes. La primera palabra clave utilizada para la búsqueda fue 'Interfaz Cerebro-Computador' tanto en español como en inglés. Los resultados obtenidos son un poco más de cien artículos y libros, decenas de Datasets (de bioseñales relacionados con intención de movimiento), varias patentes y algunos proyectos vigentes.

Sin embargo, cuando los criterios de búsqueda incluyen la palabra 'rehabilitación de miembros superiores' (o dispositivos de asistencia de miembros superiores, o exomanos, o estimulación eléctrica funcional) el número de resultados disminuye. Este número disminuye drásticamente cuando se añade 'lesiones medulares' (o paraplejia o tetraplejía) y además se filtra estos resultados en una ventana de tiempo de 10 años que va de 2007 a 2017.

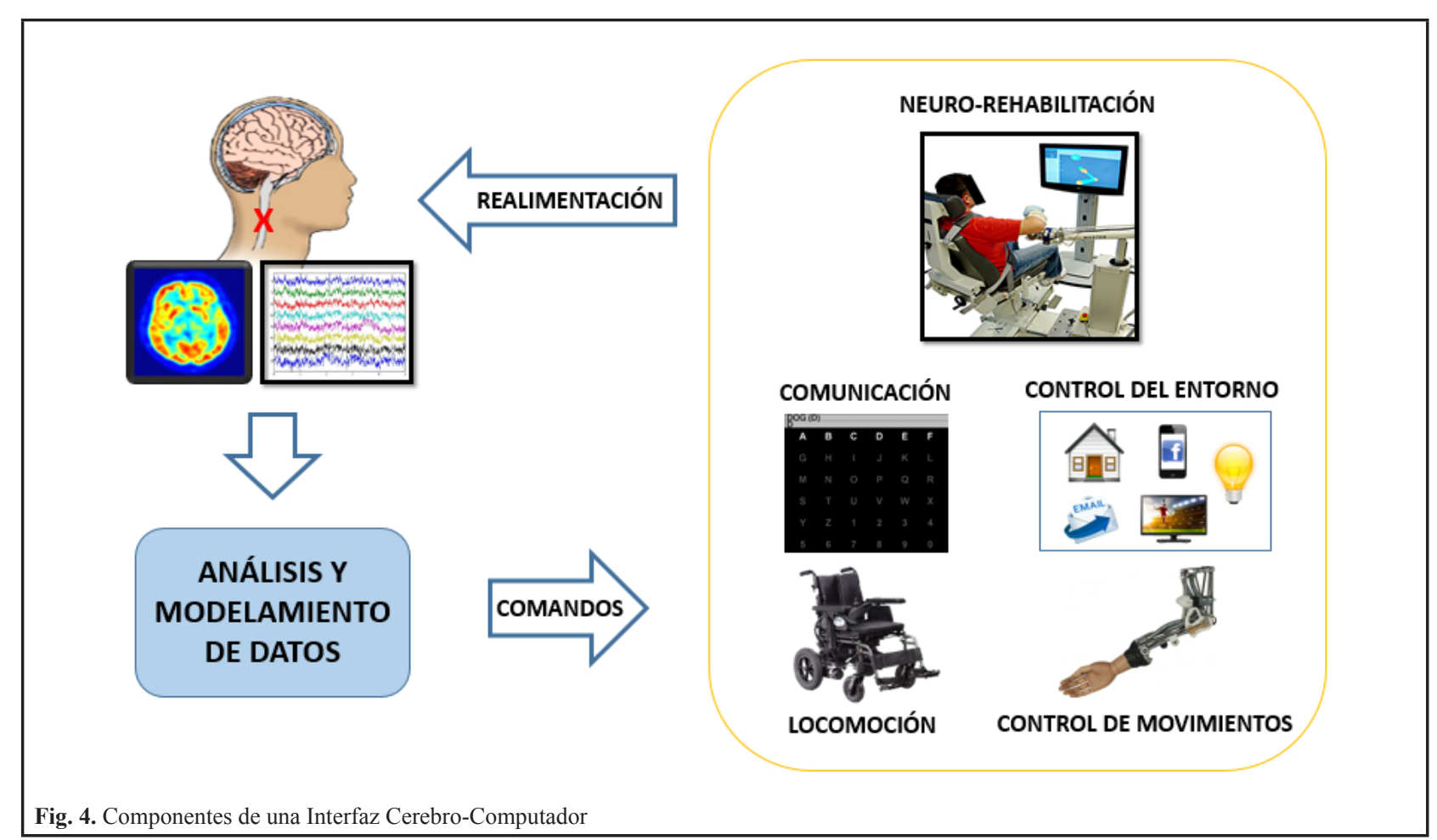




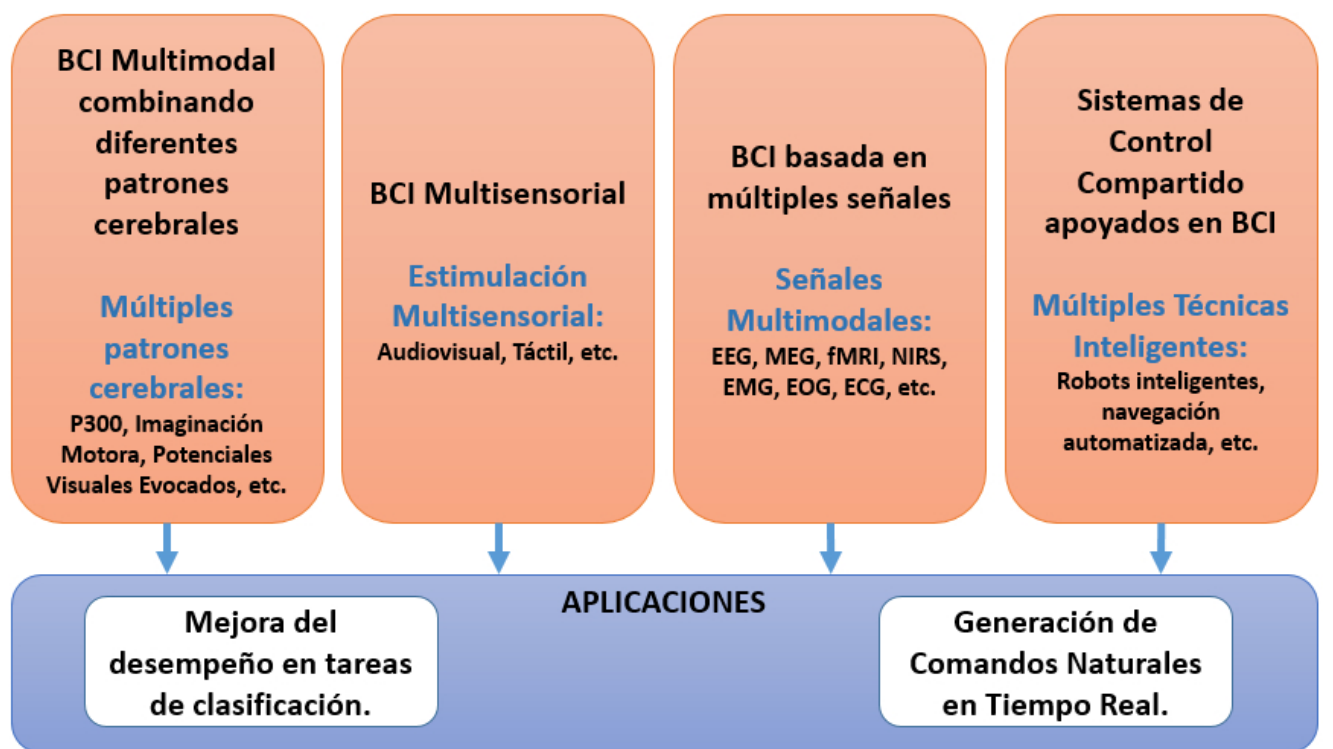

Fig. 5. Cuatro clases de Interfaces BCI Multimodal y sus aplicaciones. Adaptada de [65]

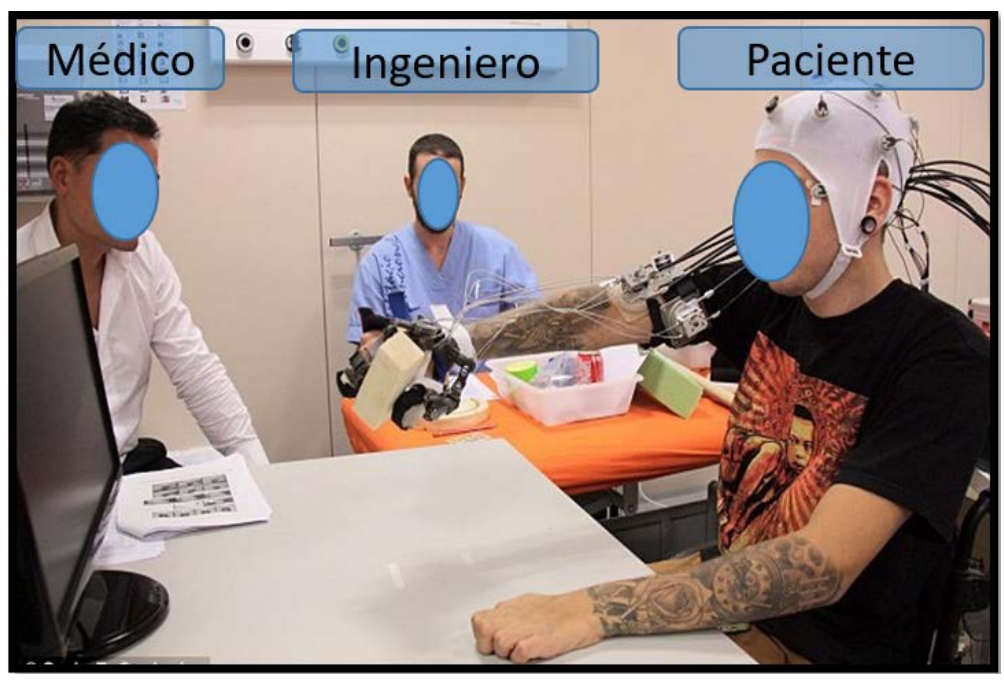

Fig. 6. Rehabilitación miembro superior con neuroprótesis mediante BCI multimodal EEG/EOG y dispositivo de asistencia de miembros superior. Tomada de [72], basado en el trabajo de investigación publicado por [12]

La mayor parte de estos resultados pertenecen a trabajos realizados en Estados Unidos y en países de Europa. Aun cuando en Latinoamérica este campo de investigación no es nuevo, a la fecha no se lograron encontrar trabajos relacionados al uso de $\mathrm{BCI}$ multimodales para procesos de rehabilitación de miembro superior en pacientes con lesión medular. Sin embargo, debe destacarse los importantes trabajos realizados por [3], alrededor de la misma temática pero a nivel de miembros inferiores en Brasil. Por otra parte, quedó demostrado que esta línea de investigación se ha potenciado gracias a los Datasets (de acceso público vía internet) ya que estos han permitido el desarrollo de mu- chas técnicas de procesado y análisis de señales para detección de intención de movimiento, en casos cuando no se dispone de pacientes o de equipos para la consecución de bioseñales asociadas a la intención de movimiento. En la actualidad, y con los criterios de búsqueda aquí utilizados, no se logró encontrar un Dataset de acceso público de bioseñales asociadas a intención de movimiento perteneciente a pacientes con lesiones medulares. Entre los Datasets más recientes basados en intención de movimiento de personas sanas se destacan los reportados en [50, 69- 71]. Estos Datasets permiten trabajar decodificación de intención de movimiento con y sin ayuda en estímulos externos. 


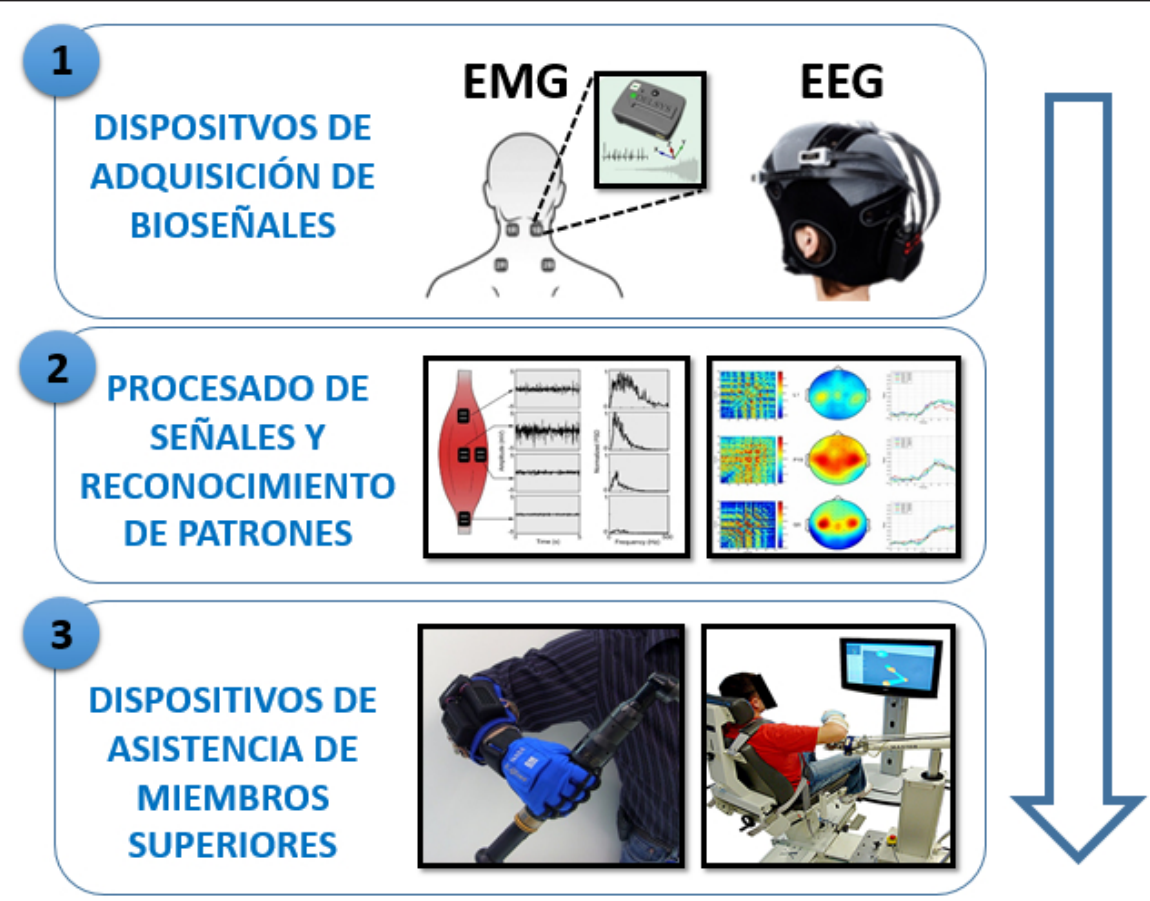

Fig. 7. BCI basada en EEG/EMG para control de dispositivos de asistencia de miembro superior para neurorrehabilitación

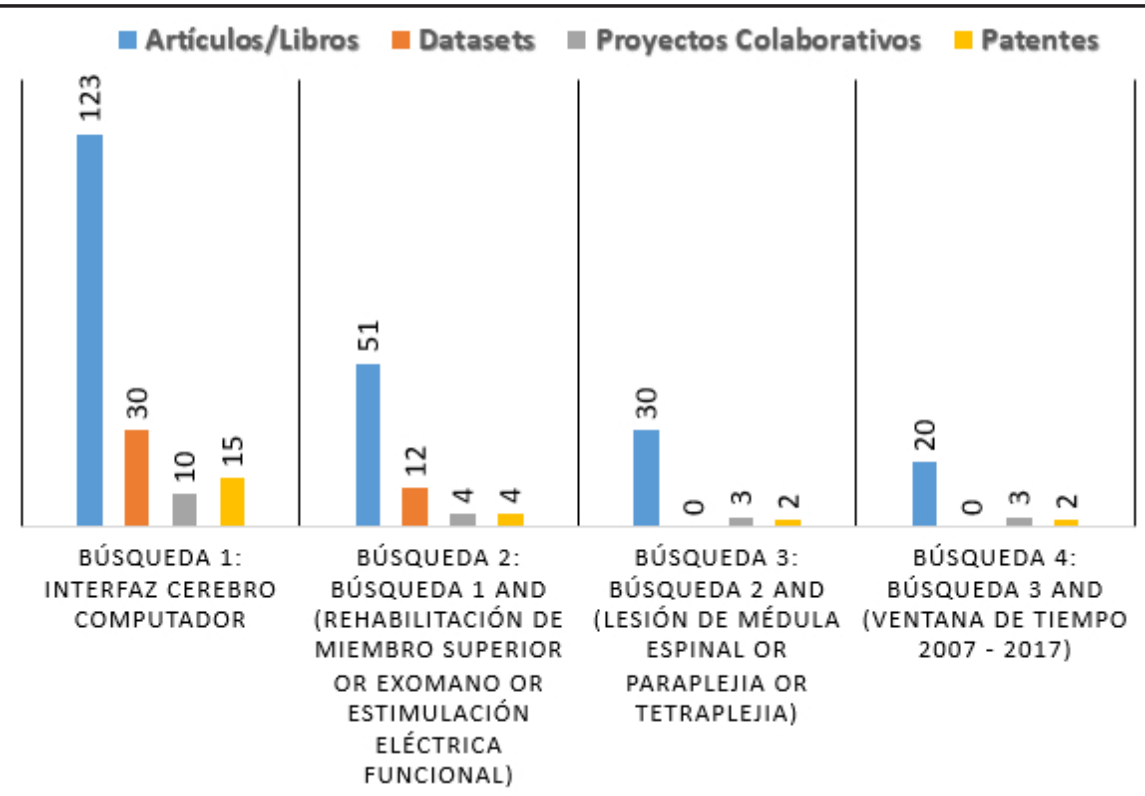

Fig. 8. Número de publicaciones por categoría (Artículo/Libros, Datasets, Proyectos Colaborativos y Patentes) utilizando criterios incrementales de búsqueda. La Búsqueda 1 es la inicial y las siguientes búsquedas añaden de forma inclusiva (operador AND) la búsqueda inmediatamente anterior

\section{Conclusiones}

La revisión de resultados de investigaciones a nivel mundial permitió evidenciar que la utilización de sistemas BCI presentan beneficios en los procesos de neurorrehabilitación de miembros superiores en pacientes con lesiones de médula espinal. Así mismo, que las investigaciones sobre BCI deben enfocarse en dos aspectos fundamentales: i) utilización de nuevos paradigmas multimodales para alcanzar altos desempeños que los conviertan en una herramienta funcional; ii) la generación de comandos naturales.

Para el caso de pacientes con lesiones medulares la elección de un tipo particular de BCI multimodal debe ser 
concertada con el especialista teniendo en cuenta la cantidad de bioseñales presentes útiles para comandar dispositivos de asistencias de miembro superior, las condiciones clínicas propias de cada paciente, y las actividades propias en los procesos de rehabilitación. El desempeño de las BCI multimodales orientadas a decodificación de intención de movimiento mejorará en la medida que pueda ofrecerse a la comunidad científica acceso público a Datasets de pacientes con lesiones medulares. Con la ayuda de estos Datasets se podrá evaluar técnicas de procesamiento de señales, reconocimiento de patrones e inclusive de visión por computador concebidas inicialmente para otras aplicaciones y que han mostrado importantes avances en desempeño. En particular, los autores de este trabajo consideran que la adecuada fusión de diferentes patrones cerebrales y patrones EMG pueden contribuir a la mejora del desempeño en la decodificación de intenciones de movimiento. Por otra parte, para la generación de comandos naturales se plantea que es necesario encontrar patrones robustos (por ejemplo, aquellos que tenga en cuenta la naturaleza no estacionaria y espacial de la señal EEG) en los potenciales CNV y BP que anteceden al inicio del movimiento; de igual forma es necesario y desarrollar mejores estrategias en las BCI de control compartido teniendo en cuenta las necesidades particulares de cada paciente en la ejecución de tareas específicas como las de alcanzar, agarrar y soltar objetos.

Para finalizar, se evidencia la gran oportunidad de investigación que esta temática ofrece a grupos de investigación multidisciplinarios: específicamente del sector de salud e ingeniería; igualmente se resalta la necesidad de potenciar este campo de investigación mediante la creación de Datasets que sean de acceso público. Sin duda, la temática aquí planteada seguirá constituyendo una pregunta abierta de investigación por mucho tiempo. Por esto, es necesario que países en vía de desarrollo como Colombia apoyen este campo de investigación el cual impactará directamente a la mejora de la calidad de vida de las personas con lesiones medulares y las de su familia.

\section{Agradecimientos}

A Colciencias, por el apoyo brindado en el marco de la convocatoria 727 del Programa de Doctorados Nacionales, 2015 y a la Vicerrectoría de Investigaciones de la Universidad del Valle por la gestión administrativa en la ejecución de la Investigación Doctoral.

\section{CONFlicto DE InTERÉS}

Los autores declaran que no tienen ningún conflicto de interés y que el manuscrito ha referenciado debidamente las ideas consignadas por otros autores.

\section{REFERENCIAS}

[1]. C. P. Henao-lema and J. E. Pérez-Parra, "Lesiones medulares y discapacidad: revisión bibliográfica," Aquichan, vol. 10, pp. 157-172, 2010.

[2]. S. Thuret, L. D. F. Moon, and F. H. Gage, "Therapeutic interventions after spinal cord injury," Nat. Rev. Neurosci., vol. 7, no. 8, pp. 628-643, Aug. 2006.

[3]. A. R. C. Donati et al., "Long-Term Training with a BrainMachine Interface-Based Gait Protocol Induces Partial Neurological Recovery in Paraplegic Patients," Sci. Rep., vol. 6, no. 6, p. 30383, Aug. 2016.

[4]. O. Tsuji et al., "Therapeutic potential of appropriately evaluated safe-induced pluripotent stem cells for spinal cord injury," Proc. Natl. Acad. Sci., vol. 107, no. 28, pp. 12704-12709, Jul. 2010.

[5]. E. A. J. Joosten, "Biodegradable biomatrices and bridging the injured spinal cord: the corticospinal tract as a proof of principle," Cell Tissue Res., vol. 349, no. 1, pp. 375-395, Jul. 2012.

[6]. V. Sahni and J. A. Kessler, "Stem cell therapies for spinal cord injury,” Nat. Rev. Neurol., vol. 6, no. 7, pp. 363-372, Jul. 2010.

[7]. S. Harkema et al., "Effect of epidural stimulation of the lumbosacral spinal cord on voluntary movement, standing, and assisted stepping after motor complete paraplegia: a case study," Lancet, vol. 377, no. 9781, pp. 1938-1947, Jun. 2011.

[8]. M. Grangeon, P. Revol, A. Guillot, G. Rode, and C. Collet, "Could motor imagery be effective in upper limb rehabilitation of individuals with spinal cord injury? A case study," Spinal Cord, vol. 50, no. 10, pp. 766-771, 2012.

[9]. E. Kandle, J. Schwartz, T. Jessel, S. Siegelbaum, and A. Hudspedth, Principles of Neural Science, 5th ed. McGraw Hill Medical, 2013.

[10]. S. C. Kirshblum et al., "Reference for the 2011 revision of the international standards for neurological classification of spinal cord injury," J. Spinal Cord Med., vol. 34, no. 6, pp. 547-554, Nov. 2011.

[11]. M. Rohm, G. R. Müller-Putz, A. Kreilinger, A. von Ascheberg, and R. Rupp, "A hybrid Brain-Computer Interface for control of a reaching and grasping neuroprosthesis," Biomed Tech, pp. 1-4, 2010.

[12]. S. R. Soekadar, M. Witkowski, N. Vitiello, and N. Birbaumer, "An EEG/EOG-based hybrid brain-neural computer interaction (BNCI) system to control an exoskeleton for the paralyzed hand," Biomed. Eng. / Biomed. Tech., vol. 60, no. 3, pp. 199-205, Jan. 2015.

[13]. J. Arata, K. Ohmoto, R. Gassert, O. Lambercy, H. Fujimoto, and I. Wada, "A new hand exoskeleton device for rehabilitation using a three-layered sliding spring mechanism," in 2013 IEEE International Conference on Robotics and Automation, 2013, pp. 3902-3907.

[14]. P. Heo, G. M. Gu, S. jin Lee, K. Rhee, and J. Kim, "Current hand exoskeleton technologies for rehabilitation and assistive engineering," Int. J. Precis. Eng. Manuf., vol. 13, no. 5, pp. 807-824, 2012.

[15]. J. D. R. Millán et al., "Combining brain-computer interfaces and assistive technologies: state-of-the-art and challenges," Front. Neurosci., vol. 1, no. SEP, pp. 1-15, 2010. 
[16]. S. J. Connolly, A. Mclntyre, S. Mehta, B. L. Foulon, and R. W. Teasell, "Upper Limb Rehabilitation Following Spinal Cord Injury," 2009.

[17]. C. P. Murphy and R. G. Chuinard, "Management of the upper extremity in traumatic tetraplegia.," Hand Clin., vol. 4, no. 2, pp. 201-9, May 1988.

[18]. A. Bryden, A. Sinnott, and M. J. Mulcahey, "Innovative Strategies for Improving Upper Extremity Function in Tetraplegia and Considerations in Measuring Functional Outcomes," Top. Spinal Cord Inj. Rehabil., vol. 10, no. 4, pp. 75-93, Apr. 2005.

[19]. L. Harvey, "Principles of conservative management for a nonorthotic tenodesis grip in tetraplegics.," J. Hand Ther, vol. 9, no. 3, pp. 238-42, 1996.

[20]. P. H. Peckham et al., "Efficacy of an implanted neuroprosthesis for restoring hand grasp in tetraplegia: A multicenter study," Arch. Phys. Med. Rehabil., vol. 82, no. 10, pp. 1380-1388, Oct. 2001.

[21]. Consortium for Spinal Cord Medicine and P. Veterans, Preservation of upper limb function following spinal cord injury: a clinical practice guideline for health-care professionals., vol. 28, no. 5. The Journal of Spinal Cord Medicine, 2005.

[22]. G. J. Snoek, M. J. IJzerman, M. W. Post, A. M. Stiggelbout, M. J. Roach, and G. Zilvold, "Choice-Based Evaluation for the Improvement of Upper-Extremity Function Compared With Other Impairments in Tetraplegia," Arch. Phys. Med. Rehabil., vol. 86, no. 8, pp. 1623-1630, Aug. 2005.

[23]. J. M. M. Hummel, G. J. Snoek, J. A. van Til, W. van Rossum, and M. J. Ijzerman, "A multicriteria decision analysis of augmentative treatment of upper limbs in persons with tetraplegia.," J. Rehabil. Res. Dev., vol. 42, no. 5, pp. 635-44, 2005.

[24]. J. Van Tuijl, Y. Janssen-Potten, and H. Seelen, "Evaluation of upper extremity motor function tests in tetraplegics," Spinal Cord, vol. 40, pp. 51-64, 2002.

[25]. M. J. Mulcahey, D. Hutchinson, and S. Kozin, "Assessment of upper limb in tetraplegia: considerations in evaluation and outcomes research.," J. Rehabil. Res. Dev., vol. 44, no. 1, pp. 91-102, 2007.

[26]. A. M. Bryden, H. A. Hoyen, M. W. Keith, M. Mejia, K. L. Kilgore, and G. A. Nemunaitis, "Upper extremity assessment in tetraplegia: The importance of differentiating between upper and lower motor neuron paralysis," Arch. Phys. Med. Rehabil., vol. 97, no. 6, pp. S97-S104, 2016.

[27]. J. H. Kaas, "Neural Plasticity," in International Encyclopedia of the Social \& Behavioral Sciences, Elsevier, 2001, pp. 10542-10546.

[28]. J. S. Seo, J. Haitsma, T. Kalker, and C. D. Yoo, “A robust image fingerprinting system using the Radon transform," Signal Process. Image Commun., vol. 19, no. 4, pp. 325-339, Apr. 2004.

[29]. K. S. Beekhuizen and E. C. Field-Fote, "Massed Practice versus Massed Practice with Stimulation: Effects on Upper Extremity Function and Cortical Plasticity in Individuals with Incomplete Cervical Spinal Cord Injury," Neurorehabil. Neural Repair, vol. 19, no. 1, pp. 33-45, Mar. 2005.

[30]. A. Curt, H. J. A. Van Hedel, D. Klaus, and V. Dietz, "Recovery from a Spinal Cord Injury: Significance of Compensation, Neural Plasticity, and Repair," J. Neurotrauma, vol. 25, no. 6, pp. 677-685, Jun. 2008.
[31]. D. Backus, "Exploring the Potential for Neural Recovery After Incomplete Tetraplegia Through Nonsurgical Interventions," $P M \& R$, vol. 2, no. 12, pp. S279-S285, Dec. 2010.

[32]. M. Alam, W. Rodrigues, B. N. Pham, and N. V. Thakor, "Brainmachine interface facilitated neurorehabilitation via spinal stimulation after spinal cord injury: Recent progress and future perspectives," Brain Res., vol. 1646, pp. 25-33, 2016.

[33].M. A. Lebedev and M. A. L. Nicolelis, "Brain-Machine Interfaces: From Basic Science to Neuroprostheses and Neurorehabilitation," Physiol. Rev., vol. 97, no. 2, pp. 767-837, 2017.

[34]. J. J. Shih, D. J. Krusienski, and J. R. Wolpaw, "Brain-Computer Interfaces in Medicine," Mayo Clin. Proc., vol. 87, no. 3, pp. 268-279, Mar. 2012.

[35]. M. Rohm et al., "Hybrid brain-computer interfaces and hybrid neuroprostheses for restoration of upper limb functions in individuals with high-level spinal cord injury," Artif. Intell. Med., vol. 59, no. 2, pp. 133-142, Oct. 2013.

[36]. W. D. Memberg et al., "Implanted Neuroprosthesis for Restoring Arm and Hand Function in People With High Level Tetraplegia," Arch. Phys. Med. Rehabil., vol. 95, no. 6, p. 1201-1211.e1, Jun. 2014.

[37]. A. Pedrocchi et al., "MUNDUS project: MUltimodal Neuroprosthesis for daily Upper limb Support," J. Neuroeng. Rehabil., vol. 10, no. 1, p. 66, 2013.

[38]. R. Arnoldina, "Neurorehabilitation and Neuroprosthetic Technologies To Regain Motor Function Following Spinal Cord Injury," ETH Zurich, 2014.

[39]. A. B. Ajiboye et al., "Restoration of reaching and grasping movements through brain-controlled muscle stimulation in a person with tetraplegia: a proof-of-concept demonstration," Lancet, vol. 389, no. 10081, pp. 1821-1830, May 2017.

[40]. A. E. Hassanien and A. T. Azar, Eds., Brain-Computer Interfaces: Current Trends and Applications, vol. 74. Cham: Springer International Publishing, 2015.

[41]. L. M. Alonso-Valerdi, R. A. Salido-Ruiz, and R. A. RamirezMendoza, "Motor imagery based brain-computer interfaces: An emerging technology to rehabilitate motor deficits," Neuropsychologia, vol. 79, pp. 354-363, Dec. 2015.

[42]. S. Ulmer and O. Jansen, Eds., fMRI: Basics and Clinical Applications. Berlin, Heidelberg: Springer Berlin Heidelberg, 2013.

[43]. B. K. Das, Ed., Positron Emission Tomography. New Delhi: Springer India, 2015.

[44]. N. Naseer and K.-S. Hong, "Classification of functional nearinfrared spectroscopy signals corresponding to the right- and left-wrist motor imagery for development of a brain-computer interface," Neurosci. Lett., vol. 553, pp. 84-89, Oct. 2013.

[45]. F. Lopes da Silva, "EEG and MEG: Relevance to Neuroscience," Neuron, vol. 80, no. 5, pp. 1112-1128, Dec. 2013.

[46]. X. Yong and C. Menon, "EEG classification of different imaginary movements within the same limb," PLoS One, vol. 10, no. 4, pp. 1-24, 2015.

[47]. C. Vidaurre, C. Klauer, T. Schauer, A. Ramos-Murguialday, and K. R. Muller, "EEG-based BCI for the linear control of an upper-limb neuroprosthesis," Med. Eng. Phys., vol. 38, no. 11, pp. 1195-1204, 2016. 
[48]. A. Shakeel, M. S. Navid, M. N. Anwar, S. Mazhar, M. Jochumsen, and I. K. Niazi, "A Review of Techniques for Detection of Movement Intention Using Movement-Related Cortical Potentials," Comput. Math. Methods Med., vol. 2015, pp. $1-13,2015$.

[49]. K. J. Miller, G. Schalk, E. E. Fetz, M. den Nijs, J. G. Ojemann, and R. P. N. Rao, "Cortical activity during motor execution, motor imagery, and imagery-based online feedback," Proc. Natl. Acad. Sci., vol. 107, no. 9, pp. 4430-4435, Mar. 2010.

[50]. P. Ofner, A. Schwarz, J. Pereira, and G. R. Müller-Putz, "Upper limb movements can be decoded from the time-domain of lowfrequency EEG," PLoS One, vol. 12, no. 8, p. e0182578, Aug. 2017.

[51]. W. G. Walter, R. Cooper, V. J. Aldridge, W. C. McCallum, and A. L. Winter, "Contingent Negative Variation: An Electric Sign of Sensori-Motor Association and Expectancy in the Human Brain," Nature, vol. 203, no. 4943, pp. 380-384, Jul. 1964.

[52]. H. H. Kornhuber and L. Deecke, "Hirnpotentialänderungen bei Willkürbewegungen und passiven Bewegungen des Menschen: Bereitschaftspotential und reafferente Potentiale," Pflüger's Arch. für die gesamte Physiol. des Menschen und der Tiere, vol. 284, no. 1, pp. 1-17, 1965.

[53]. O. F. do Nascimento, K. D. Nielsen, and M. Voigt, "Movementrelated parameters modulate cortical activity during imaginary isometric plantar-flexions," Exp. Brain Res., vol. 171, no. 1, pp. 78-90, May 2006.

[54]. J. Jin, B. Allison, Y. Zhang, X. Wang, and A. Cichocki, “An ERPBased BCI Using and Oddbal Paradigm with Different Faces And Reduced Errors in Critical Functions," Int. J. Neural Syst., vol. 24, no. 8, p. 1450027, Dec. 2014.

[55]. A. Barachant, S. Bonnet, M. Congedo, and C. Jutten, "Classification of covariance matrices using a Riemannian-based kernel for BCI applications," Neurocomputing, vol. 112, pp. 172-178, 2013.

[56]. M. Congedo, A. Barachant, and A. Andreev, "A New Generation of Brain-Computer Interface Based on Riemannian Geometry," arXiv Prepr. arXiv1310.8115, vol. 33, no. 0, Oct. 2013.

[57]. S. Mingxu, "A FES Control System for Upper Limb Rehabilitation," University of Salford, 2014.

[58]. I. Systems and A. Technology, "A Study of Controlling UpperLimb Exoskeletons Using EMG and EEG signals," Saga University, 2014.

[59]. E. López-Larraz, L. Montesano, Á. Gil-Agudo, and J. Minguez, "Continuous decoding of movement intention of upper limb self-initiated analytic movements from pre-movement EEG correlates," J. Neuroeng. Rehabil., vol. 11, no. 1, p. 153, 2014.

[60]. A. Schwarz, P. Ofner, J. Pereira, A. I. Sburlea, and G. R. MüllerPutz, "Decoding natural reach-and-grasp actions from human EEG," J. Neural Eng., vol. 26, no. 10, p. 105037, Aug. 2017.

[61]. D. Liu, W. Chen, R. Chavarriaga, Z. Pei, and J. del R. Millán, "Decoding of Self-paced Lower-Limb Movement Intention: A Case Study on the Influence Factors," Front. Hum. Neurosci., vol. 11, no. November, pp. 1-12, Nov. 2017.

[62]. L. Randazzo, I. Iturrate, R. Chavarriaga, R. Leeb, and J. R. Del Millan, "Detecting intention to grasp during reaching movements from EEG," in 2015 37th Annual International Conference of the IEEE Engineering in Medicine and Biology Society (EMBC), 2015, vol. 2015-Novem, pp. 1115-1118.
[63]. E. Lew, R. Chavarriaga, S. Silvoni, and J. Millán, "Detection of self-paced reaching movement intention from EEG signals," Front. Neuroeng., vol. 5, no. July, pp. 1-17, 2012.

[64]. L. Yang, H. Leung, D. A. Peterson, T. J. Sejnowski, and H. Poizner, "Toward a Semi-Self-Paced EEG Brain-Computer Interface: Decoding Initiation State from Non-Initiation State in Dedicated Time Slots," PLoS One, vol. 9, no. 2, p. e88915, Feb. 2014.

[65].Y. Li et al., "Multimodal BCIs: Target Detection, Multidimensional Control, and Awareness Evaluation in Patients with Disorder of Consciousness," Proc. IEEE, vol. 104, no. 2, pp. 332-352, 2016.

[66]. J. Arcos-Argoty, E. Cossio-García, and R. Villa-Torres, "Evaluación experimental y estadística de un prototipo de intefaz cerebro-computadora (ICC)," Rev. Ing. Biomédica, vol. 4, no. 8, pp. 22-33, 2013.

[67]. P. A. Vélez-Ángel, H. Villafañe-Saldarriaga, and H. LoaizaCorrea, "Clasificación Básica de Neuroseñales," Universidad Tecnológica de Pereira, 2010.

[68]. D. A. Reyes-Lopez, M. Arias-López, J. E. Duarte-Sánchez, and H. Loaiza-Correa, "Implementación en FPGA de un clasificador de movimientos de la mano usando señales EMG," Redes Ing., vol. 6, no. 1, p. 85, Sep. 2015.

[69]. M. D. Luciw, E. Jarocka, and B. B. Edin, "Multi-channel EEG recordings during 3,936 grasp and lift trials with varying weight and friction," Sci. Data, vol. 1, pp. 1-11, 2014.

[70]. D. Steyrl, R. Scherer, J. Faller, and G. R. Müller-Putz, "Random forests in non-invasive sensorimotor rhythm brain-computer interfaces: A practical and convenient non-linear classifier," Biomed. Tech., vol. 61, no. 1, pp. 77-86, 2016.

[71]. J. D. Wander et al., "Cortico-Cortical Interactions during Acquisition and Use of a Neuroprosthetic Skill," PLoS Comput. Biol., vol. 12, no. 8, pp. 1-20, 2016.

[72]. R. O'Hare, “The mind-reading robotic hand that lets quadriplegic people grasp everyday objects," Spinal Cord Injury Zone, 2016. [Online]. Available: http://www.spinalcordinjuryzone.com/ news/17563/mind-reading-robotic-hand-lets-quadriplegic-peoplegrasp-everyday-objects. [Accessed: 03-Feb-2017]. 